\title{
Real-World Treatment of Complicated Skin and Soft Tissue Infections with Daptomycin: Results from a Large European Registry (EU-CORE)
}

\author{
Alberto Cogo • Armando Gonzalez-Ruiz • Rashidkhan Pathan • \\ Kamal Hamed (D)
}

To view enhanced content go to www.infectiousdiseases-open.com

Received: June 1, 2015 / Published online: July 14, 2015

(c) The Author(s) 2015. This article is published with open access at Springerlink.com

\section{ABSTRACT}

Introduction: The objective of this analysis was to describe in real-life settings the clinical outcomes and safety associated with daptomycin treatment in a cohort of patients with complicated skin and soft tissues infection (cSSTI).

Methods: All patients with cSSTI who had received at least one dose of daptomycin between January 2006 and April 2012 were identified from a non-interventional, multicenter, retrospective registry (European

Electronic supplementary material The online version of this article (doi:10.1007/s40121-015-0074-x) contains supplementary material, which is available to authorized users.

\section{A. Cogo}

Casa Di Cura Villa Berica, U.O. Medicina Vascolare e

Piede Diabetico, Vicenza, Italy

A. Gonzalez-Ruiz

Darent Valley Hospital, Dartford, UK

R. Pathan

Novartis Healthcare Pvt. Ltd., Hyderabad, India

\section{K. Hamed ( $\square)$}

Novartis Pharmaceuticals Corporation,

East Hanover, NJ, USA

e-mail: kamal.hamed@novartis.com
Cubicin ${ }^{\circledR}$ Outcome Registry and Experience; $\mathrm{EU}_{-\mathrm{CORE}}{ }^{\mathrm{SM}}$ ).

Results: Of the 6075 patients included in the EU-CORE registry, 1927 (31.7\%) were diagnosed with cSSTI (male, 63.8\%; median age, 63 years). The most frequent underlying diseases were cardiovascular disease (58.1\%) and diabetes mellitus (40.7\%). The most frequent cSSTIs included surgical site infections (34.9\%), wound infections (20.2\%) and diabetic foot infections (19.9\%). The most frequently prescribed doses of daptomycin were $4 \mathrm{mg} / \mathrm{kg} /$ day (38.9\%) and $6 \mathrm{mg} / \mathrm{kg} /$ day $(35.2 \%)$. A total of 1126 (58.4\%) patients received antibiotics prior to daptomycin treatment; treatment failure $(53.7 \%)$ was the most common reason for switching to daptomycin. The majority of hospitalized patients (61.8\%) were treated with concomitant antibiotics. Among patients with positive cultures, Staphylococcus aureus (51.9\%; 673/1297) was the most common pathogen. The overall clinical success rate was $84.6 \%$; for infections caused by $S$. aureus, the success rate was $87.2 \%$ (methicillin susceptible, $87.8 \%$; methicillin resistant, 87.0\%). Adverse events possibly related to daptomycin treatment were reported 
in $2.4 \%$ of patients and adverse events led to drug discontinuation in $2.4 \%$ of patients.

Conclusion: Daptomycin treatment resulted in high clinical success rates in patients with different cSSTI subtypes, the majority of whom having failed previous antibiotic therapy. Daptomycin was well tolerated and there were no new or unexpected safety findings.

Keywords: Complicated skin and soft tissue infection; Daptomycin; EU-CORE; Gram-positive infections; Staphylococci

\section{INTRODUCTION}

Skin and soft tissue infections (SSTIs) are the consequence of a microbial invasion of the layers of the skin and underlying soft tissues [1]. Complicated SSTIs (cSSTIs) represent more severe cases that involve deeper soft tissues and often require surgical treatment (e.g., infected ulcers, burns or major abscesses) [2]. Moreover, associated comorbidities and microbial factors, particularly drug resistance, make the treatment of cSSTI more challenging [3].

Staphylococcus aureus is the main causative pathogen of cSSTIs [3]. Further, methicillinresistant S. aureus (MRSA) complicates their treatment. The percentages of MRSA isolates reported in Europe range from $0.7 \%$ (Sweden) to 53.9\% (Romania) [4]. Prolonged hospital stay, economic burden and significant morbidity are associated with cSSTI due to MRSA [5].

The glycopeptide vancomycin has been widely used for the treatment of MRSA infections, and its overuse has resulted in the emergence of vancomycin-intermediate and vancomycin-resistant $S$. aureus [6]. As a consequence, the increase of the minimum inhibitory concentrations (MICs) of vancomycin for MRSA is now a major challenge [7, 8]. Increased relapse rates have been reported for MRSA infections with a high vancomycin MIC [9].

Early therapy with anti-MRSA agents in cSSTI reduces in-hospital mortality, shortens length of hospital stay and decreases treatment cost $[10,11]$. Therefore, the initial antibiotic treatment of cSSTI is frequently empirical before identification of the causative pathogen. Empirical coverage of both methicillin-susceptible $S$. aureus (MSSA) and MRSA infections is important, because failure of initial antibiotic therapy in cSSTI is significantly associated with worse clinical and economic outcomes [12].

Daptomycin, a cyclic lipopeptide, displays rapid bactericidal activity against a wide range of Gram-positive pathogens including pathogens resistant to methicillin and vancomycin [13]. Daptomycin is approved for the treatment of cSSTI $(4 \mathrm{mg} / \mathrm{kg} /$ day $)$, rightsided infective endocarditis due to $S$. aureus and bacteremia associated with cSSTI or right-sided infective endocarditis (6 mg/kg/day) [14]. In two pivotal trials, the clinical success rate in patients with cSSTI was $83.4 \%$ [15]. Of interest, daptomycin required a shorter duration of treatment than comparator antibiotics chosen by the study investigators [15].

The European Cubicin ${ }^{\circledR}$ Outcomes Registry and Experience (EU-CORE ${ }^{\mathrm{SM}}$ ) collected data to evaluate daptomycin therapy in a real-world setting. The results from this registry during the first 2.5 years of clinical experience with inpatients with cSSTI or bacteremia have been published previously [16]. The present analysis describes the final overall results of the EUCORE registry (2006-2012) on the effectiveness and safety of daptomycin in the subgroup of patients with cSSTI. 


\section{METHODS}

\section{Patients and Data Collection}

EU-CORE was a non-interventional, multicenter, retrospective registry designed to collect real-world outcome data from patients who had received at least one dose of daptomycin for the treatment of a Grampositive bacterial infection between January 2006 and April 2012 with at least 30 days of safety follow-up. The study was performed across 18 countries in Europe $(n=12)$, Latin America $(n=5)$, and Asia $(n=1)$. The data were collected by local investigators using standardized case report forms. Patients who might have received daptomycin as part of a controlled clinical trial were excluded from the study. Details on methods of EU-CORE have been described elsewhere [17]. For the present study, patients with cSSTI were selected and analyzed for treatment outcome and safety. An interim analysis of 373 patients with cSSTI selected from the first EU-CORE reporting period has been published (1127 patients enrolled between January 2006 and August 2008) [16].

The protocol was approved by the health authority and the Institutional Review Board (IRB) or Ethics Committee (EC) in each country and written informed consent was obtained according to the requirements of the IRB or EC and/or the local data privacy regulations.

\section{Clinical Outcomes and Safety}

Clinical outcomes at the end of daptomycin therapy were assessed for each of the patients included in the registry using the following protocol-defined criteria: cured, clinical signs and symptoms resolved, no additional antibiotic therapy was necessary, or infection cleared with a negative culture reported; improved, partial resolution of clinical signs and symptoms and/or additional antibiotic therapy was warranted; failed, inadequate response to daptomycin therapy, worsening or new/recurrent signs and symptoms, need for a change in antibiotic therapy, or a positive culture reported at the end of therapy; and non-evaluable, unable to determine response because of insufficient information. Clinical success was defined as patients who were cured or improved. The time to improvement was also recorded. The reasons for stopping daptomycin therapy and other antibiotics prescribed following daptomycin were also collected.

Safety assessments included reports of adverse events (AEs) and serious AEs (SAEs) up to 30 days after the end of daptomycin treatment along with their intensity and relationship to daptomycin. All AEs, regardless of seriousness or severity, were recorded.

\section{Statistical Analysis}

The safety population included all eligible patients with at least one safety assessment and the efficacy population included all eligible patients for whom clinical outcome was assessed.

Owing to the nature of the trial, inferential analyses were not performed and no formal statistical methodology other than simple descriptive statistics was used. Continuous variables were summarized as arithmetic mean, standard deviation, median and range; categorical variables were summarized by absolute and relative frequencies.

Statistical analysis was performed using SAS version 9.3 (SAS Institute Inc., Cary, NC, USA). 


\section{RESULTS}

\section{Patient Demographic and Clinical Characteristics}

Of the 6075 daptomycin-treated patients included in the EU-CORE registry, 1927 $(31.7 \%)$ were diagnosed with cSSTI. Daptomycin therapy was completed without further antibiotic treatment for 1196 (62.1\%) patients; 443 (23.0\%) patients switched from daptomycin therapy (e.g., step-down to oral antibiotic therapy) and the remaining 288 (14.9\%) patients discontinued treatment due to various reasons (AEs, $n=47$ [2.4\%]; treatment failure, $n=53 \quad[2.8 \%]$; unable to determine, $n=66[3.4 \%] ;$ and other or missing/unknown reasons, $n=122$ [6.3\%]).

Baseline demographics and clinical characteristics of these patients are summarized in Table 1 . The majority of patients were male (63.8\%, $n=1230)$ and had a median age of 63.0 years (range 1-103). The most frequent underlying diseases were cardiovascular disease (58.1\%, $n=1119)$ and diabetes mellitus (40.7\%, $n=784)$. The most frequent cSSTIs were surgical site infections (SSIs; 34.9\%, $n=673$ ), wound infections $(20.2 \%, n=389)$, and diabetic foot infections $(19.9 \%, n=383)$.

\section{Microbiology}

Culture results were available for 82.0\% (1580/ 1927 ) of patients; culture was positive for $82.1 \%$ $(1297 / 1580)$ of them. The most frequent primary pathogens isolated from patients with cSSTI were $S$. aureus $(51.9 \%, n=673)$ and to a lesser degree coagulase-negative Staphylococcus (CoNS) (17.5\%, $\quad n=227), \quad$ including Staphylococcus epidermidis (10.9\%, $n=141$; Table 2). MRSA was reported in $31.5 \%$
Table 1 Demographic and clinical characteristics

\begin{tabular}{|c|c|}
\hline Characteristics & $\begin{array}{l}N=1927 \\
n(\%)\end{array}$ \\
\hline Age $^{\mathrm{a}}$ (years), median (range) & $63(1-103)$ \\
\hline$<65$ years & $1007(52.3)$ \\
\hline$\geq 65$ years & $919(47.7)$ \\
\hline$\geq 75$ years & $443(23.0)$ \\
\hline Gender, male & $1230(63.8)$ \\
\hline Ethnicity $^{\mathrm{b}}$, Caucasian & $1713(88.9)$ \\
\hline Body weight (kg), median (range) & $77.0(7-200)$ \\
\hline \multicolumn{2}{|l|}{ Significant underlying diseases $(>5 \%)^{c}$} \\
\hline Cardiovascular disease & $1119(58.1)$ \\
\hline Diabetes mellitus & $784(40.7)$ \\
\hline Renal disease & $278(14.4)$ \\
\hline Pulmonary disease & $226(11.7)$ \\
\hline Oncologic disease & $197(10.2)$ \\
\hline Gastrointestinal disease & $174(9.0)$ \\
\hline \multicolumn{2}{|l|}{ Complicated skin and soft tissue infections } \\
\hline Surgical site infection & $673(34.9)$ \\
\hline Superficial incision & $236(12.2)$ \\
\hline Deep incision & $298(15.5)$ \\
\hline Organ/space & $139(7.2)$ \\
\hline Wound & $389(20.2)$ \\
\hline Diabetic foot & $383(19.9)$ \\
\hline Deep ulcer & $189(9.8)$ \\
\hline Major abscess & $186(9.7)$ \\
\hline $\begin{array}{l}\text { Diabetic ulcer (excluding diabetic foot } \\
\text { infections) }\end{array}$ & $107(5.6)$ \\
\hline
\end{tabular}

Results are given as $n(\%)$ unless otherwise stated

a Missing data for $n=1$

b Missing data for $n=34$

c Patients may have one or more underlying disease

$(n=408)$ of patients, whereas MSSA was isolated in $16.4 \%(n=213)$ of patients with cSSTI. 
Table 2 Primary pathogens

\begin{tabular}{lc}
\hline Culture results and primary pathogens & $\begin{array}{l}\boldsymbol{N}=\mathbf{1 9 2 7} \\
\text { isolated }\end{array}$ \\
\hline Available culture & $1580(82.0)$ \\
Negative culture & $283(17.9)$ \\
Positive culture & $1297(82.1)$ \\
Staphylococcus aureus & $673(51.9)$ \\
Methicillin resistant & $408(31.5)$ \\
Methicillin susceptible & $213(16.4)$ \\
Methicillin susceptibility unknown & $52(4.0)$ \\
Coagulase-negative staphylococci & $227(17.5)$ \\
Staphylococcus epidermidis & $141(10.9)$ \\
Methicillin resistant & $15(1.2)$ \\
Methicillin susceptible & $103(7.9)$ \\
Methicillin susceptibility unknown & $23(1.8)$ \\
Other & $86(6.6)$ \\
Methicillin resistant & $12(0.9)$ \\
Methicillin susceptible & $55(4.2)$ \\
Methicillin susceptibility unknown & $19(1.5)$ \\
Enterococcus faecalis & $71(5.5)$ \\
Vancomycin resistant & $6(0.5)$ \\
Non-vancomycin resistant & $48(3.7)$ \\
Vancomycin susceptibility unknown & $17(1.3)$ \\
Enterococcus faecium & $54(4.2)$ \\
Vancomycin resistant & $9(0.7)$ \\
Non-vancomycin resistant & $36(2.8)$ \\
Vancomycin susceptibility unknown & $9(0.7)$ \\
Enterococcus species & $13(1.0)$ \\
\hline & $259(20.0)$ \\
\hline
\end{tabular}

${ }^{a}$ Other includes Clostridium perfringens, Corynebacterium spp., Gram-positive cocci, Peptostreptococcus spp., Staphylococcus spp. (coagulase not specified), Streptococcus agalactiae or group B streptococci, Streptococcus dysgalactiae, Streptococcus dysgalactiae equisimilis, Streptococcus pyogenes or group A streptococci, Streptococcus spp., viridans streptococci, Gram-negative bacilli, Gram-positive bacilli, Gram-negative cocci, fungi/ yeast, and invalid/ambiguous pathogen code

\section{Previous and Concomitant Antibiotic Therapy}

Antibiotics prior to daptomycin therapy were received by $58.4 \%(n=1126)$ of patients with cSSTI. Treatment failure $(53.7 \%, n=1034)$ was the most common reason for switching to daptomycin therapy, with failure most commonly occurring in patients treated with penicillins (11.9\%, $n=229)$, fluoroquinolones (8.0\%, $\quad n=154)$, cephalosporins $\quad(7.0 \%$, $n=134)$, or glycopeptides $(6.6 \%, n=127)$. Concomitant antibiotics were given more frequently to hospitalized patients (61.8\%, $n=1190)$ than to outpatients $(3.3 \%, n=63)$. The most common concomitant antibiotics were carbapenems $(22.9 \%, n=442)$ and fluoroquinolones $(15.6 \%, n=301)$.

\section{Daptomycin Prescribing Patterns}

Daptomycin was prescribed as a first-line therapy in $34.2 \%(n=659)$ of patients and as second-line therapy in $58.4 \%(n=1126)$ of patients. The commonly prescribed doses of daptomycin in patients with cSSTI were $4 \mathrm{mg} / \mathrm{kg} /$ day $\quad(38.9 \%, \quad n=750) \quad$ and $6 \mathrm{mg} / \mathrm{kg} /$ day $(35.2 \%, n=678)$. Of the 408 patients with MRSA infection, $32.1 \%$ ( $n=131$ were treated with $4 \mathrm{mg} / \mathrm{kg} /$ day and $30.6 \%(n=125)$ with $6 \mathrm{mg} / \mathrm{kg} /$ day. However, among the 62 patients with MSSA infection, the most common dose of daptomycin was $6 \mathrm{mg} / \mathrm{kg} /$ day (59.7\%), followed by $4 \mathrm{mg} / \mathrm{kg} /$ day $\quad$ (25.8\%). Higher doses $(>6 \mathrm{mg} / \mathrm{kg} /$ day and $\leq 10 \mathrm{mg} / \mathrm{kg} /$ day) were received by $11.1 \% \quad(n=214)$ of patients and $0.2 \% \quad(n=3)$ of patients received doses $>10 \mathrm{mg} / \mathrm{kg} /$ day.

The total median duration of therapy in patients with cSSTI was 11 days with a range of 1-210 days [10.0 days for outpatients (range 
1-189 days); 10.0 days for in-patients (range 1-137 days) and 8.0 days for intensive care patients (range 1-90 days)].

\section{Clinical Outcomes}

Clinical success rates were high for all doses. The overall clinical success rate (cured or improved $)$ was $84.7 \% \quad(n=1632) ; \quad 6.1 \%$ $(n=118)$ patients had treatment failure, and 9.2\% $(n=177)$ patients were non-evaluable. High clinical success rates were observed in all subtypes of cSSTI. The clinical success rates for cSSTI subtypes were highest for diabetic ulcer infections (91.6\%) and superficial incision SSIs (88.1\%; Fig. 1). Overall, clinical success rates by primary pathogen were comparable as detailed in Fig. 2.

The median time to improvement in patients with CSSTI was 4 days in patients receiving a daptomycin dose of $4 \mathrm{mg} / \mathrm{kg} /$ day (range 1-37) or $6 \mathrm{mg} / \mathrm{kg} /$ day (range $1-41$ ).

\section{Safety}

Overall, $10.5 \%(n=203)$ of patients reported AEs including 2.4\% $(n=46)$ with AEs possibly related to daptomycin (Table 3). Daptomycin was discontinued due to an AE for $2.4 \%(n=47)$ of patients.

Increased blood creatine phosphokinase (CPK) was reported for $1.3 \% \quad(n=26)$ of patients and multi-organ failure for $1.2 \%$ $(n=24)$. SAEs were reported for $5.9 \%(n=114)$ of patients, of whom $0.6 \%(n=12)$ with SAEs possibly related to daptomycin.

\section{DISCUSSION}

EU-CORE was a retrospective, multicenter registry, designed to collect real-world data on the use of daptomycin for Gram-positive infections. The results of the current analysis provide greater insights into the prescribing patterns of daptomycin and outcomes in cSSTI patients. In particular, a large proportion of the cSSTI population of EU-CORE was elderly and included patients with poor clinical conditions at baseline. Nevertheless, daptomycin treatment resulted in high clinical success rates in patients with different types of CSSTI, the majority of whom not responding to prior antibiotic therapies. As expected, the most frequently reported causative agent was S. aureus followed by CoNS. Overall, the present final analysis on 1927 patients of the cSSTI population of EU-CORE confirms the previous interim analysis on 373 patients with cSSTI [16].

A majority of patients received either $4 \mathrm{mg} / \mathrm{kg} /$ day (38.9\%), the approved dose of daptomycin for cSSTI without bacteremia, or $6 \mathrm{mg} / \mathrm{kg} /$ day (35.2\%), which is approved for cSSTI with bacteremia. Doses $>6 \mathrm{mg} / \mathrm{kg} /$ day were received by $11.1 \%$ of patients. These high doses could reflect treatment of patients with serious or deep-seated cSSTIs (e.g., diabetic foot infection, major abscess). Moreover, recent guidelines recommend the use of high doses of daptomycin (from 8 to $10 \mathrm{mg} / \mathrm{kg} /$ day) in patients with moderate-tosevere or severe MRSA SSTIs associated with ischemia [18].

The high clinical success rate (84.7\%) observed in the present EU-CORE study is in line with the results observed in the CORE study [19]. These results are also consistent with previously reported randomized clinical trials which assessed the efficacy of daptomycin in patients with cSSTI $[15,20]$. In the two pivotal studies of Arbeit et al. [15], the clinical success rate was $83.4 \%(372 / 446)$. In a randomized Phase IIIb trial, Konychev et al. [20] observed a clinical success of $89.0 \%(65 / 73)$ in elderly 


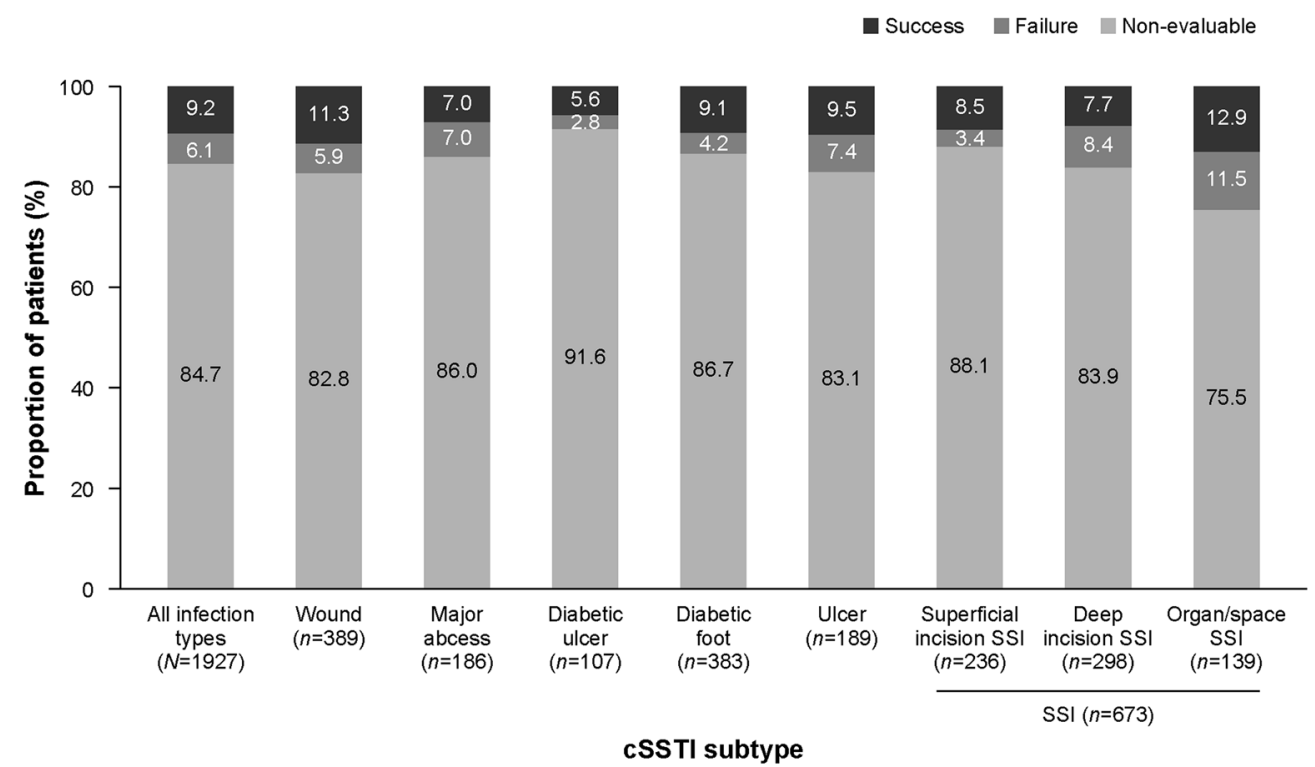

Fig. 1 Clinical outcome by cSSTI subtype. cSSTI complicated skin and soft tissue infection, SSI surgical site infection

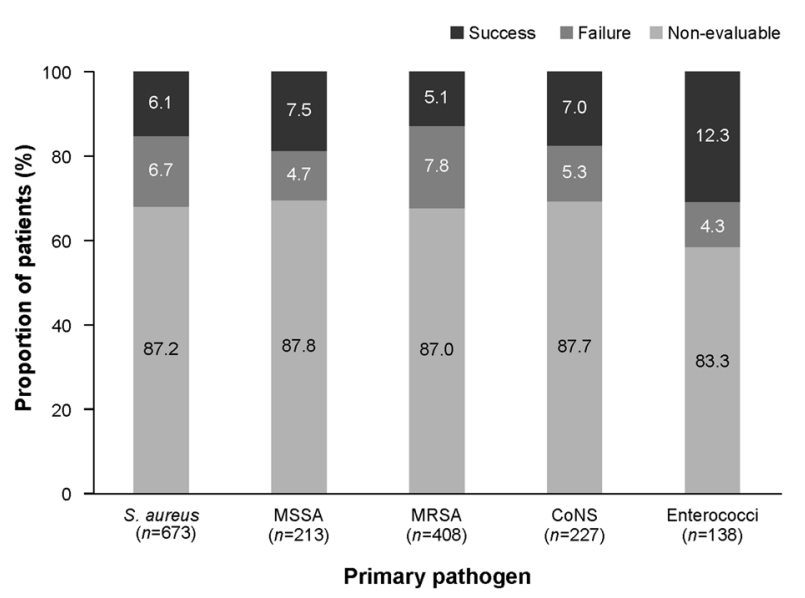

Fig. 2 Clinical outcome by predominant primary pathogen. CoNS coagulase-negative Staphylococcus, MRSA methicillin-resistant Staphylococcus aureus, MSSA methicillin-susceptible $S$. aureus

patients ( $\geq 65$ years) receiving daptomycin as first-line treatment for cSSTI. Quist et al. [11] reported a clinical success of $91.4 \%(53 / 58)$ in a randomized clinical trial in patients with firstline treatment of daptomycin. In the metaanalysis of Wang et al. [21] that included six randomized controlled trials, the overall clinical success in patients with cSSTI was 85.5\% (630/ 737). Therefore, these results further support the use of daptomycin as a first-line treatment option for cSSTI.

Our results confirmed in real-world settings a comparable high effectiveness across infections caused by various pathogens, in particular MRSA. These results support the use of daptomycin as empiric treatment for cSSTI. Indeed, $S$. aureus is the most frequent pathogen causing cSSTIs and it is advantageous to start treatment with antibiotics that are effective against both MSSA and MRSA in settings with high MRSA rates. Early treatment of cSSTI caused by MRSA was associated with lower in-hospital mortality rates, shorter length of hospital stay and lower total costs [10].

The current analysis displayed high clinical success rates in different types of cSSTI including diabetic foot infection, which is a serious, difficult-to-treat infection [18]. These results support previous results from Joseph et al. who showed that success in patients with diabetic foot infection treated with daptomycin was higher in patients who had surgery and when initial therapy was 
Table 3 Summary of safety results of daptomycin treatment

\begin{tabular}{lc}
\hline Safety parameters & $\begin{array}{l}N=1927 \\
n(\%)\end{array}$ \\
\hline AEs & $203(10.5)$ \\
SAEs & $114(5.9)$ \\
Discontinuations due to AEs & $47(2.4)$ \\
AEs possibly related to daptomycin treatment & $46(2.4)$ \\
Skin and subcutaneous tissue disorders & $12(0.6)$ \\
General disorders and administration site & $8(0.4)$ \\
$\quad$ conditions & $7(0.4)$ \\
Investigations & $7(0.4)$ \\
Blood CPK increased & $2(0.1)$ \\
Musculoskeletal and connective tissue & $12(0.6)$ \\
$\quad$ disorders & \\
Myalgia &
\end{tabular}

$A E$ adverse event, $C P K$ creatine phosphokinase, $S A E$ serious $\mathrm{AE}$

daptomycin [22]. In the exploratory study of Knapp et al. on the use of daptomycin in SSIs in 30 patients with superficial incision SSI and 30 patients with deep SSI, the clinical success rates were $100 \%$ and $92 \%$, respectively [23].

Daptomycin showed a favorable safety profile in this patient population without any new or unexpected safety findings. Study drug discontinuations due to AEs were infrequent. The blood CPK elevations were minimal.

The limitations of the present analysis were first related to the non-randomized and retrospective design of the study. Moreover, the treatment outcome was judged by the local investigators who were not blinded. No central laboratory was used and results of primary pathogens and their susceptibility could be different across the different centers. Another limitation is that many of the cSSTIs are mixed infections of both Gram-positive and Gramnegative bacteria. Daptomycin being specific for Gram-positive bacteria, the mixed cSSTI might not be cured completely, thus affecting the clinical success rate, and also increasing the need for concomitant antibiotic therapy. There was also no restriction on the use of concomitant antibiotics. Although the use of concomitant antibiotics complicated the data interpretation for both clinical outcome and safety of daptomycin, the treatment outcome associated with daptomycin reflected its use in real life. Despite these limitations, high levels of success were reported in the different centers that included patients in the registry with no stringent criteria. Indeed, the only criterion was daptomycin treatment for cSSTI. Therefore, patients with poor conditions who would have been excluded in a randomized trial were included in the registry.

\section{CONCLUSIONS}

Daptomycin treatment resulted in high clinical success rates in patients with different types of cSSTI, the majority of whom failed previous antibiotic therapy. Daptomycin was well tolerated and there were no new or unexpected safety findings.

\section{ACKNOWLEDGMENTS}

This study and article processing charges were sponsored by Novartis Pharma AG. All named 
authors meet the International Committee of Medical Journal Editors (ICMJE) criteria for authorship for this manuscript, take responsibility for the integrity of the work as a whole, and have given final approval for the version to be published. Medical writing support was provided by Novartis Global Medical and Clinical Services medical writers Sai Krishnaveni Chevooru and Stephen Sullivan. Funding for writing was provided by Novartis Pharma AG.

Conflict of interest. Alberto Cogo has nothing to disclose. Armando Gonzalez-Ruiz received fees from Novartis, Pfizer, Cubist, and Gilead for staff training, being member of Advisory Boards and being member of speakers panels. He also received support to attend scientific conferences including accommodation and travel payments, and a Novartis grant to support his research. Rashidkhan Pathan is an employee of Novartis healthcare Pvt. Ltd. Kamal Hamed is an employee of Novartis Pharmaceuticals Corporation.

Compliance with ethics guidelines. The study has been performed in accordance with the ethical standards as laid down in the 1964 Declaration of Helsinki and its later amendments or comparable ethical standards. The protocol was approved by the health authority and the Institutional Review Board (IRB) or Ethics Committee (EC) in each country and written informed consent was obtained according to the requirements of the IRB or EC and/or the local data privacy regulations.

Open Access. This article is distributed under the terms of the Creative Commons Attribution Noncommercial License which permits any noncommercial use, distribution, and reproduction in any medium, provided the original author(s) and the source are credited.

\section{REFERENCES}

1. Ki V, Rotstein C. Bacterial skin and soft tissue infections in adults: a review of their epidemiology, pathogenesis, diagnosis, treatment and site of care. Can J Infect Dis Med Microbiol. 2008;19:173-84.

2. Dryden MS. Complicated skin and soft tissue infection. J Antimicrob Chemother. 2010;65(Suppl 3):iii35-44.

3. Bassetti M, Baguneid M, Bouza E, Dryden M, Nathwani D, Wilcox M. European perspective and update on the management of complicated skin and soft tissue infections due to methicillinresistant Staphylococcus aureus after more than 10 years of experience with linezolid. Clin Microbiol Infect. 2014;20(Suppl 4):3-18.

4. European Centre for Disease Prevention and Control. Antimicrobial resistance surveillance in Europe 2012. Annual Report of the European Antimicrobial Resistance Surveillance Network (EARS-Net). Stockholm: ECDC; 2013.

5. Athanasakis K, Petrakis I, Ollandezos M, et al. Antibacterial treatment of meticillin-resistant Staphylococcus aureus complicated skin and soft tissue infections: a cost and budget impact analysis in Greek hospitals. Infect Dis Ther. 2014;3:257-68.

6. Loomba PS, Taneja J, Mishra B. Methicillin and vancomycin resistant $S$. aureus in hospitalized patients. J Glob Infect Dis. 2010;2:275-83.

7. Sievert DM, Rudrik JT, Patel JB, McDonald LC, Wilkins MJ, Hageman JC. Vancomycin-resistant Staphylococcus aureus in the United States, 2002-2006. Clin Infect Dis. 2008;46:668-74.

8. Steinkraus G, White R, Friedrich L. Vancomycin MIC creep in non-vancomycin-intermediate Staphylococcus aureus (VISA), vancomycinsusceptible clinical methicillin-resistant $S$. aureus (MRSA) blood isolates from 2001-05. J Antimicrob Chemother. 2007;60:788-94.

9. Choi EY, Huh JW, Lim CM, et al. Relationship between the MIC of vancomycin and clinical outcome in patients with MRSA nosocomial pneumonia. Intensive Care Med. 2011;37:639-47.

10. Itani KM, Akhras KS, Stellhorn R, Quintana A, Budd D, Merchant S. Outcomes associated with initial 
versus later vancomycin use in patients with complicated skin and skin-structure infections. Pharmacoeconomics. 2009;27:421-30.

11. Quist SR, Fierlbeck G, Seaton RA, Loeffler J, Chaves RL. Comparative randomised clinical trial against glycopeptides supports the use of daptomycin as first-line treatment of complicated skin and softtissue infections. Int $\mathrm{J}$ Antimicrob Agents. 2012;39:90-1.

12. Edelsberg J, Berger A, Weber DJ, Mallick R, Kuznik A, Oster G. Clinical and economic consequences of failure of initial antibiotic therapy for hospitalized patients with complicated skin and skin-structure infections. Infect Control Hosp Epidemiol. 2008;29:160-9.

13. Rybak MJ. The efficacy and safety of daptomycin: first in a new class of antibiotics for Gram-positive bacteria. Clin Microbiol Infect. 2006;12(Suppl $1): 24-32$.

14. Novartis Europharm Ltd. Cubicin ${ }^{\circledR}$ (daptomycin) EU Summary of Product Characteristics; 2012.

15. Arbeit RD, Maki D, Tally FP, Campanaro E, Eisenstein BI. The safety and efficacy of daptomycin for the treatment of complicated skin and skin-structure infections. Clin Infect Dis. 2004;38:1673-81.

16. Gonzalez-Ruiz A, Beiras-Fernandez A, Lehmkuhl H, Dohmen PM, Loeffler J, Chaves RL. Effectiveness and safety of daptomycin in complicated skin and soft-tissue infections and bacteraemia in clinical practice: results of a large non-interventional study. Int J Antimicrob Agents. 2013;41:372-8.
17. Gonzalez-Ruiz A, Beiras-Fernandez A, Lehmkuhl H, Seaton RA, Loeffler J, Chaves RL. Clinical experience with daptomycin in Europe: the first 2.5 years. J Antimicrob Chemother. 2011;66:912-9.

18. Blanes JI. Consensus document on treatment of infections in diabetic foot. Rev Esp Quimioter. 2011;24:233-62.

19. Martone WJ, Lamp KC. Efficacy of daptomycin in complicated skin and skin-structure infections due to methicillin-sensitive and -resistant Staphylococcus aureus: results from the CORE Registry. Curr Med Res Opin. 2006;22:2337-43.

20. Konychev A, Heep M, Moritz RK, et al. Safety and efficacy of daptomycin as first-line treatment for complicated skin and soft tissue infections in elderly patients: an open-label, multicentre, randomized phase IIIb trial. Drugs Aging. 2013;30:829-36.

21. Wang SZ, Hu JT, Zhang C, et al. The safety and efficacy of daptomycin versus other antibiotics for skin and soft-tissue infections: a meta-analysis of randomised controlled trials. BMJ Open. 2014;4:e004744.

22. Joseph WS, Quast T, Cogo A, et al. Daptomycin for methicillin-resistant Staphylococcus aureus diabetic foot infections. $\mathrm{J}$ Am Podiatr Med Assoc. 2014;104:159-68.

23. Knapp AG, Kamepalli RK, Martone WJ, Yankelev S. Prospective, non-comparative study of daptomycin for the treatment of superficial and deep incisional surgical site infections. Surg Infect (Larchmt). 2011;12:113-8. 